\title{
Infection Control Preparedness for Human Infection With Influenza A H7N9 in Hong Kong
}

\author{
Vincent C. C. Cheng, MD, FRCPath; ${ }^{1,2}$ Josepha W. M. Tai, PhD; ${ }^{2}$ W. M. Lee, MsN; ${ }^{2}$ W. M. Chan, MBBS, FRCP; ${ }^{3}$ \\ Sally C. Y. Wong, MBBChir, MRCP; ${ }^{1}$ Jonathan H. K. Chen, PhD; ${ }^{1}$ Rosana W. S. Poon, PhD; ${ }^{1}$ \\ Kelvin K. W. To, MBBS, FRCPath; ${ }^{1}$ Jasper F. W. Chan, MBBS, FRCPath; ${ }^{1}$ P. L. Ho, MD, FRCPath; ${ }^{1}$ \\ K. H. Chan, PhD; ${ }^{1}$ K. Y. Yuen, MD, FRCPath ${ }^{1}$
}

ов јестіve. To assess the effectiveness of infection control preparedness for human infection with influenza A H7N9 in Hong Kong.

DESIGN. A descriptive study of responses to the emergence of influenza A H7N9.

SETting. A university-affiliated teaching hospital.

PARTICIPANTS. Healthcare workers (HCWs) with unprotected exposure (not wearing N95 respirator during aerosol-generating procedure) to a patient with influenza A H7N9.

METHODs. A bundle approach including active and enhanced surveillance, early airborne infection isolation, rapid molecular diagnostic testing, and extensive contact tracing for HCWs with unprotected exposure was implemented. Seventy HCWs with unprotected exposure to an index case were interviewed especially regarding their patient care activities.

Results. From April 1, 2013, through May 31, 2014, a total of 126 (0.08\%) of 163,456 admitted patients were tested for the H7 gene by reverse transcription-polymerase chain reaction per protocol. Two confirmed cases were identified. Seventy (53.8\%) of 130 HCWs had unprotected exposure to an index case, whereas $41(58.6 \%)$ and $58(82.9 \%)$ of 70 HCWs wore surgical masks and practiced hand hygiene after patient care, respectively. Sixteen $(22.9 \%)$ of 70 HCWs were involved in high-risk patient contacts. More HCWs with high-risk patient contacts received oseltamivir prophylaxis $(P=0.088)$ and significantly more had paired sera collected for $\mathrm{H} 7$ antibody testing $(P<0.001)$. Ten $(14.3 \%)$ of 70 HCWs developed influenza-like illness during medical surveillance, but none had positive results by reverse transcriptionpolymerase chain reaction. Paired sera was available from 33 of 70 HCWs with unprotected exposure, and none showed seroconversion against H7N9.

CONCLUsions. Despite the delay in airborne precautions implementation, no patient-to-HCW transmission of influenza A H7N9 was demonstrated.

Infect Control Hosp Epidemiol 2015;36(1):87-92

\section{N T RO D U C T I O N}

The emergence of novel influenza A H7N9 in March 2013 posed a public health threat locally and internationally. ${ }^{1-3}$ The pathogenicity of influenza A H7N9 appeared to be lower than that of $\mathrm{H} 5 \mathrm{~N} 1$, but the former was capable of replicating in the upper and lower respiratory tract. ${ }^{4}$ Limited airborne transmission of influenza A H7N9 has been demonstrated between ferrets, ${ }^{5}$ and intrafamilial spread of this virus has been reported ${ }^{6-9}$ hence it is important to intensify the infection control preparedness and minimize the risk of nosocomial transmission of influenza A H7N9. Here, we present our proactive infection control measures against influenza A H7N9 in Hong Kong.

\section{METHODS}

\section{Infection Control Preparedness for Influenza A H7N9}

Specific proactive infection control measures were established in Queen Mary Hospital, a university-affiliated hospital of 1,600 beds, on April 1, 2013, to prevent nosocomial transmission of influenza A H7N9. Active and enhanced surveillance was performed according to the clinical and epidemiologic criteria to identify suspected cases presented to our hospital (Table 1). Patients recruited under active surveillance were provided with a bundle of care, including early segregation in an airborne infection isolation facility for contact, droplet, and contact precautions; collection of respiratory specimens for a rapid molecular

Affiliations: 1. Department of Microbiology, Queen Mary Hospital, Hong Kong, China; 2. Infection Control Team, Queen Mary Hospital, Hong Kong, China; 3. Department of Adult Intensive Care Unit, Queen Mary Hospital, Hong Kong, China.

Received June 24, 2014; accepted August 15, 2014

(c) 2015 by The Society for Healthcare Epidemiology of America. All rights reserved. 0899-823X/2015/3601-0013. DOI: 10.1017/ice.2014.2 
тавLE 1. Clinical and Epidemiologic Criteria for Active and Enhanced Surveillance of Influenza A H7N9 in Hong Kong ${ }^{\mathrm{a}}$

Active surveillance

\begin{tabular}{ll}
\hline A. & Clinical criteria \\
1. & Patient with influenza-like illness (temperature $>38^{\circ} \mathrm{C}$ with cough or sore throat); or \\
2. & Patient with severe pneumonia; or \\
3. & Patient died of unexplained acute respiratory illness \\
B. & Epidemiologic criteria \\
1. & Contact with a human case of influenza A H7N9; or \\
2. & Contact with poultry or wild birds or their remains or with environments contaminated by their feces in countries/areas with \\
3. & in poultry and/or humans in the previous 6 months; or \\
4. & Close contact with a confirmed influenza A H7N9-infected animal other than poultry or wild birds; or \\
5. & Worked in a laboratory that is processing samples from persons or animals that are suspected from avian influenza infection.
\end{tabular}

Enhanced surveillance

\begin{tabular}{|c|c|}
\hline A. & Clinical criteria \\
\hline 1. & $\begin{array}{l}\text { Community-acquired pneumonia with unknown causes, which is not responding to antimicrobial treatment for typical and } \\
\text { atypical agents for } 3 \text { days; or }\end{array}$ \\
\hline 2. & Community-acquired pneumonia requiring intensive care support \\
\hline B. & Epidemiologic criteria \\
\hline 1. & Community-acquired pneumonia occurring in cluster; or \\
\hline 2. & Community-acquired pneumonia occurring in healthcare workers; or \\
\hline
\end{tabular}

${ }^{a}$ For active surveillance, patients fulfilling any options in both clinical and epidemiologic criteria were tested for influenza A M gene and H7 specific HA genes by reverse transcription-polymerase chain reaction; whereas for enhanced surveillance, patients fulfilling either clinical or epidemiologic criteria were tested for influenza A M gene and $\mathrm{H} 7$ specific HA genes by reverse transcription-polymerase chain reaction.

${ }^{\mathrm{b}}$ Epidemiologic criteria include one or more of the following exposures in the 7 days (subsequently increased to 10 days on April 25, 2013) prior to symptoms onset.

${ }^{c}$ This condition was included in the epidemiologic criteria on December 19, 2013, after confirmation of the first imported case of human infection of influenza A H7N9 in Hong Kong.

diagnostic test; and notification to the Department of Health. With the increasing number of influenza A H7N9 human infections reported in mainland China, enhanced surveillance was implemented as a safety net for infected patients who were not identified during active surveillance (Table 1). Enhanced infection control measures were enforced after confirmation of the first imported case of influenza A H7N9 in Hong Kong (Table 2).

Open staff forums held in a lecture room and face-to-face bedside education sessions were arranged by the infection control team to provide "right on time" infection control updates to the frontline healthcare workers (HCWs). Hand hygiene compliance assessments were conducted regularly in our hospital. ${ }^{10}$

\section{Investigation for Possible Nosocomial Transmission of Influenza A H7N9}

Upon laboratory confirmation of a human case of influenza A H7N9, the infection control team identified HCWs and patients with unprotected exposure. Unprotected staff exposure was defined as contact within 1 meter with a confirmed case and neither of them wearing surgical masks, in a clinical setting not involving aerosol-generating procedures. If aerosol-generating procedures, as defined in Table 2, were performed during contact, unprotected exposure was defined as exposed HCWs not wearing full personal protective equipment. Unprotected patient exposure was defined as patients staying in the same cubicle in a general ward as the index patient, regardless of the duration of exposure. The list of persons who had visited the index case or other patients staying in the same cubicle was submitted to Department of Health for community follow-up.

Contact tracing was performed to identify all potentially exposed HCWs and patients in our hospital. Oseltamivir (75 mg twice daily for 5 days) was offered as postexposure prophylaxis for HCWs and patients with unprotected exposure. Healthcare workers underwent medical surveillance for 20 days ( 2 incubation periods of influenza A H7N9), with daily monitoring of body temperature and influenza-like illness by the infection control team. During medical surveillance, they were advised to wear a surgical mask in the hospital and community. Patients with unprotected exposure were quarantined for 10 days in the hospital or in the premises provided by the Department of Health if medical care was no longer required, followed by medical surveillance for another 10 days. 
T A в LE 2. Enhanced Infection Control Measures After Confirmation of Imported Case of Influenza A H7N9 in Hong Konga

Infection control measures in all clinical areas

1. Surgical mask was required for all healthcare workers and visitors in all clinical areas, including waiting areas of outpatient clinics and emergency departments.

2. Full personal protective equipment, including N95 respirator, eye protection, gloves, and gowns, was required for healthcare workers performing routine patient care practices in the high-risk areas. ${ }^{\text {b }}$

3. When aerosol-generating procedures ${ }^{\mathfrak{c}}$ were performed, patients were transferred into negative pressure airborne infection isolation facilities and healthcare workers were required to wear full personal protective equipment during the procedures.

${ }^{\mathrm{a}}$ In addition to the usual practice of hand hygiene and environmental cleanliness, infection control measures in general and high-risk clinical areas were enhanced after confirmation of the first imported case of influenza A H7N9 in Hong Kong on December 2, 2013.

${ }^{\mathrm{b}}$ High-risk areas included triage stations of outpatient clinics and emergency departments, and isolation rooms.

${ }^{c}$ Aerosol-generating procedures included endotracheal intubation, cardiopulmonary resuscitation, bronchoscopy, and open suction of respiratory tract, sputum induction, use of nebulizer therapy, noninvasive positive pressure ventilation, and high-frequency oscillatory ventilation.

Serologic testing for influenza A H7N9 was offered to HCWs with unprotected exposure. Baseline serum and convalescent serum at day 21 after exposure were collected, on a voluntary basis, to look for asymptomatic seroconversion. For HCWs with protected exposure, oseltamivir was not given but they underwent medical surveillance for 20 days.

\section{Laboratory Diagnosis}

Respiratory specimens including nasopharyngeal aspirates, sputum, endotracheal aspirates, or bronchoalveolar lavage were collected for direct immunofluorescent antigen test for influenza $\mathrm{A}$, and reverse transcription-polymerase chain reaction (RT-PCR) for influenza A M gene, H1, H3, H5, and H7 as previously described. ${ }^{11-13}$ RT-PCR runs were performed thrice daily at 11:00 AM, 4:00 PM, and midnight. A confirmed case of influenza A H7N9 was defined as a patient with positive RT-PCR results for $\mathrm{M}$ gene and $\mathrm{H7}$ specific HA gene in the respiratory specimens.

For serologic diagnosis of influenza A H7N9, serum samples were treated with receptor-destroying enzyme (1:3) (Denka Seiken) at $37^{\circ} \mathrm{C}$ overnight to remove nonspecific inhibitors, and residual receptor-destroying enzyme was destroyed by heat inactivation at $56^{\circ} \mathrm{C}$ for 30 minutes as recently described. ${ }^{14}$ The hemagglutination inhibition test was carried out in 96-well microtiter plates using reference H7N9 antigen (A/Anhui/1/2013). Serial 2-fold dilutions of receptordestroying enzyme-treated serum from 1:10 were titrated against 4 hemagglutinin units of reference antigens by incubation at room temperature for 1 hour, followed by adding $0.5 \%$ turkey erythrocytes to the serum-virus mixture and further incubation at room temperature for 30 minutes.

\section{Statistical Analysis}

The Fisher exact test was used to compare independent categorical variables between groups. All reported $P$ values were 2sided. A $P$ value of less than 0.05 was considered statistically significant. Computation was performed using SPSS, version 15.0 (IBM).

\section{RESULTS}

\section{Infection Control Preparedness for Influenza A H7N9}

From April 1, 2013, through May 31, 2014, a total of 163,456 patients were admitted to Queen Mary Hospital, of whom 126 $(0.08 \%)$ were tested for $\mathrm{H} 7$ gene by RT-PCR (Figure 1$)$. There were 80 males and 46 females. The median age was 57 years (range, 1-92 years). Twenty-two (17.5\%) of the 126 patients were younger than 15 years. One $(0.8 \%)$ of the 126 patients had positive $\mathrm{H} 7$ results by RT-PCR. Additionally, another case of influenza A H7N9, referred from a regional hospital, was managed in Queen Mary Hospital.

A total of 10 sessions of open staff forum, with an overall 1,532 hospital staff attendance, were held to update the medical knowledge of infection control concerning influenza A H7N9. Video demonstrations of gowning and degowning of personal protective equipment were provided for all isolation wards and were also uploaded to the hospital intranet for review by frontline HCWs. A bedside audit of gowning and degowning by infection control nurses was performed to ascertain compliance. Hand hygiene compliance demonstrated an increase in all clinical departments from 23\% (2007 baseline) to $66 \%$ (2010) and to $75 \%$ (2013).

\section{Investigation for Possible Nosocomial Transmission of Influenza A H7N9}

Extensive contact tracing of exposed HCWs and patients was performed according to our infection control protocol. The first case of influenza A H7N9 was in a 36-year-old Indonesian woman. The clinical presentation, viral load profile, phylogenetic analysis, and treatment of this patient has been described previously. ${ }^{12,15}$ She was transferred to the adult intensive care unit, Queen Mary Hospital, at 6:34 PM November 30, 2013, with a false-negative result for influenza $\mathrm{A}$ by direct antigen detection. She was nursed in a single-patient cubicle with no negative pressure upon arrival and received mechanical ventilation, followed by extracorporeal membrane oxygenation, together with noninvasive ventilator support. Her positive result for influenza A H7N9 by RT-PCR test was available from 


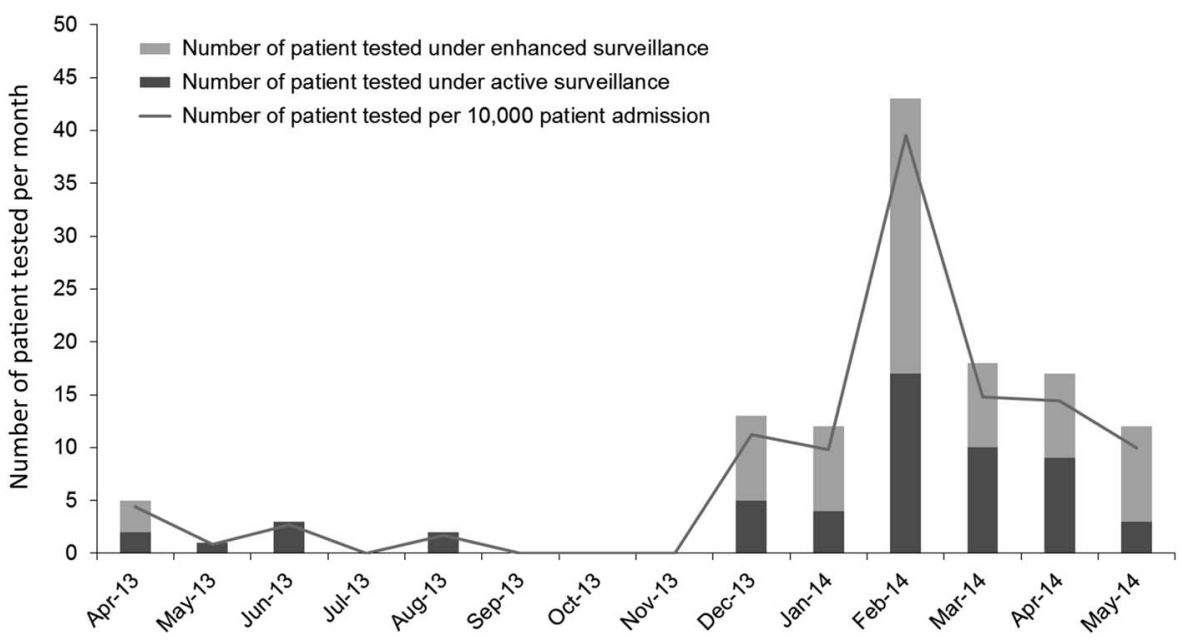

FIG URE 1. Surveillance of Influenza A H7N9 in Queen Mary Hospital from April 1, 2013, through May 31, 2014.

Fifty-six (44.4\%) of 126 patients fulfilled the clinical and epidemiologic criteria of active surveillance and were admitted directly to the airborne infection isolation rooms in internal medicine (32 patients), pediatrics (18), and adult intensive care units (6). Nasopharyngeal aspirates were the most commonly collected respiratory specimens in 54 patients, whereas endotracheal aspirates and bronchoalveolar lavage were collected in 1 patient each. None of these 56 patients had positive results for the $\mathrm{H} 7$ gene by reverse transcription-polymerase chain reaction. Seventy $(55.6 \%)$ of 126 patients were tested according to the criteria of enhanced surveillance, of which 37 patients were referred from adult intensive care unit, 26 patients from medical specialty, 3 from pediatrics, 3 from surgery, and 1 from orthopedic surgery. Nasopharyngeal aspirates were collected in 56 patients whereas lower respiratory specimens, including endotracheal aspirates and bronchoalveolar lavage, were collected in the remaining 14 patients. The median interval from admission to respiratory specimen collection was 1 day (range, 0 [on the day of admission] to 38 days).

the referral hospital after 48 hours of stay in our adult intensive care unit. The patient was immediately transferred to the airborne infection isolation room. Three debriefing sessions were held to explain the logistics of postexposure antiviral prophylaxis, collection of paired sera for $\mathrm{H} 7$ antibody test, and medical surveillance to the 70 HCWs with unprotected exposure, because none of them had worn an N95 respirator during patient care activities with aerosol-generating procedures. Forty-eight HCWs had baseline blood collected; however, only $33(47.1 \%)$ of the $70 \mathrm{HCWs}$ had paired sera available for H7 antibody analysis. Nonetheless, 15 of the 16 HCWs with unprotected exposure involving high-risk patient contact were tested and showed no seroconversion. The demographic characteristics of the $70 \mathrm{HCWs}$ under medical surveillance, with respect to high-risk and non-high-risk patient contacts, are illustrated in Table 3.

The second patient was a 65 -year-old man with chronic renal failure who had traveled to Shenzhen, mainland China, on January 1, 2014. He presented with fever and cough 3 days later and was admitted to our general medical ward via the emergency department with community-acquired pneumonia 4 days after onset of symptoms. Although this patient had no history of poultry contact, he was screened for influenza A H7N9 in the enhanced surveillance scheme (Table 1). The patient was immediately transferred to the airborne infection isolation room when the positive influenza A H7N9 RT-PCR result became available after 30 hours of hospitalization.
No aerosol-generating procedure was performed in the general ward. Contact tracing revealed 4 patients with unprotected exposure. Nasopharyngeal aspirates were collected to exclude asymptomatic infection of influenza A H7N9, and the 4 patients were quarantined for 10 days, followed by another 10 days of medical surveillance. A total of $51 \mathrm{HCWs}$ had contact with this patient or had attended the implicated cubicles at the emergency department or the general medical ward. They were considered to have protected exposure because they had worn surgical masks in the clinical areas, hence oseltamivir was not offered. During medical surveillance, 6 HCWs (11.8\%) developed influenza-like illness. They all had negative test results for H7 HA gene PCR.

\section{DISCUSSION}

When the first case of influenza A H7N9 was reported to the World Health Organization on March 31, 2014, proactive infection control measures with a bundle approach for early recognition of the index case, isolation in an airborne infection isolation room, and extensive contact tracing for HCWs and patients with unprotected exposure was immediately instituted in our hospital. Frontline HCWs in the triage station of the emergency department were responsible for identifying patients fulfilling the clinical and epidemiologic criteria of influenza A H7N9 among emergency admissions, whereas medical and nursing staff in the admission wards were responsible 
TA BLE 3. Demographic Characteristics of 70 Healthcare Workers Under Medical Surveillance After Unprotected Exposure to a Confirmed Case of Influenza A H7N9 for 48 Hours

\begin{tabular}{|c|c|c|c|}
\hline & $\begin{array}{l}\text { High-risk patient contacts }{ }^{\mathrm{a}} \\
\qquad(\mathrm{n}=16)\end{array}$ & $\begin{array}{l}\text { Non-high-risk patient contacts }{ }^{\mathrm{b}} \\
\qquad(\mathrm{n}=54)\end{array}$ & $P$ value \\
\hline Staff rank & & & 0.001 \\
\hline Nursing staff & $14(88 \%)$ & $27(50 \%)$ & \\
\hline Allied health staff & $2(13 \%)$ & 0 & \\
\hline Supporting staff & 0 & $14(26 \%)$ & \\
\hline Wearing of surgical mask & $13(81 \%)$ & $28(52 \%)$ & 0.036 \\
\hline Wearing of gloves & $2(13 \%)$ & $8(15 \%)$ & 0.816 \\
\hline Wearing of gowns & $2(13 \%)$ & $5(9 \%)$ & 0.704 \\
\hline Hand hygiene after patient contact & $16(100 \%)$ & $42(78 \%)$ & 0.038 \\
\hline Received oseltamivir postexposure prophylaxis & $11(69 \%)$ & $24(44 \%)$ & 0.088 \\
\hline
\end{tabular}

NOTE. ILI, influenza-like illness; NA, not applicable.

${ }^{a}$ High-risk patient contacts include disconnection of ventilator circuit for end tidal carbon dioxide monitoring in 6 staff, caring for noninvasive ventilation in 5 , extubation in 3 , and performing chest physiotherapy in 2 .

${ }^{\mathrm{b}}$ Non-high-risk patient contacts include adjustment and monitoring of medical equipment in 29 staff, patient turning and bathing in 13 , physical examination in 10 , and room cleansing in 2 .

for collecting this information among elective admitted patients as part of the active surveillance program. None of the 56 patients recruited in the active surveillance program had positive results for influenza A H7N9. In contrast, 1 of 70 patients tested for $\mathrm{H} 7$ gene under enhanced surveillance was confirmed to have influenza A H7N9. Enhanced surveillance appeared to be an important safety net in the detection of influenza A H7N9 infection because only 55\% and $67 \%$ of the confirmed patients had a history of poultry contact or had visited a live poultry market, respectively, as illustrated in the recent case control study on risk factors in patients with H7N9 disease. $^{16}$

Despite the implementation of active and enhanced surveillance, diagnosis in one of the confirmed cases was delayed owing to a high false-negative rate of the direct antigen test compared with RT-PCR. ${ }^{17}$ Delay in the diagnosis of influenza A H7N9 infection resulted in unprotected exposure of 70 HCWs. Since most of them complied with standard and droplet precautions by wearing a surgical mask and performing hand hygiene after high-risk patient contacts, none of the tested paired sera showed H7N9 antibody seroconversion. We suspect that the high hand hygiene compliance in our hospital, as a result of continuous implementation of proactive control measures against various viruses and multidrug-resistant organisms, ${ }^{11,18-22}$ has protected our frontline HCWs against many challenges of emerging and reemerging infectious diseases. Additionally, our findings suggested that use of standard and droplet precautions appeared to be effective in the prevention of nosocomial transmission of influenza A H7N9, even during the initial phase of infection where the viral load was highest. ${ }^{15}$
Our findings also concurred with the recent observation of a lack of subclinical infections among $126 \mathrm{HCW}$ s by means of serologic investigation in Zhejiang Province, China. ${ }^{23}$ Although the risk of human-to-human transmission of influenza A H7N9 virus appeared to be low, Chinese national guidelines recommend a combination of standard, contact, and droplet precautions by HCWs in the hospitals. ${ }^{9}$ The Centers for Disease Control and Prevention in the United States recommends a higher level of infection control precautions with addition of airborne precautions, in view of the lack of a widely available, safe, and effective vaccine as well as a high rate of morbidity and mortality among H7N9-infected patients. ${ }^{24}$ In Hong Kong, we adopted the Centers for Disease Control and Prevention recommendation. To circumvent the problem with limited isolation facilities in our locality, our laboratory conducts regular RT-PCR testing for all suspected cases every 8 hours, which facilitates early discharge of suspected patients from airborne infection isolation rooms when test results are negative.

There are several limitations to this study. First, only 33 (47.1\%) of the 70 HCWs with unprotected exposure had paired sera examination for H7N9 seroconversion. Subclinical infection may be underestimated. However, since almost all HCWs with unprotected exposure during high-risk patient contacts did not show seroconversion, it is unlikely that nosocomial transmission of influenza went unrecognized. Second, there is no evidence of efficient human-to-human transmission of influenza A H7N9 in the community at this stage. The lack of nosocomial transmission of influenza A H7N9 in our healthcare setting may represent the intrinsic characteristic of the virus instead of the result of our infection 
control intervention. However, compared with the higher rate of $\mathrm{H} 5$ antibody seroconversion among HCWs in Hong Kong during the outbreak of influenza A H5N1 in $1997,{ }^{25}$ our healthcare system appears to be better prepared for challenges posed by known or unknown emerging pathogens.

\section{ACKNOW LEDGMENTS}

Financial support. This work was supported by the commissioned research grant from the Health and Medical Research Fund of the Food and Health Bureau, Hong Kong Special Administrative Region.

Potential conflicts of interest. All authors report no conflicts of interest relevant to this article.

Address correspondence to K. Y. Yuen, MD, FRCPath, Department of Microbiology, Queen Mary Hospital, Hong Kong, China (kyyuen@hkucc.hku.hk).

\section{REFERENCES}

1. Gao R, Cao B, Hu Y, et al. Human infection with a novel avianorigin influenza A (H7N9) virus. N Engl J Med 2013;368:1888-1897.

2. Chen Y, Liang W, Yang S, et al. Human infections with the emerging avian influenza A H7N9 virus from wet market poultry: clinical analysis and characterisation of viral genome. Lancet 2013;381:1916-1925.

3. Meng Z, Han R, Hu Y, et al. Possible pandemic threat from new reassortment of influenza A(H7N9) virus in China. Euro Surveill, 19:20699.

4. Xu L, Bao L, Deng W, et al. Novel avian-origin human influenza $\mathrm{A}(\mathrm{H} 7 \mathrm{~N} 9)$ can be transmitted between ferrets via respiratory droplets. J Infect Dis 2014;209:551-556.

5. Richard M, Schrauwen EJ, de Graaf M, et al. Limited airborne transmission of H7N9 influenza A virus between ferrets. Nature 2013;501:560-563.

6. Jie Z, Xie J, He Z, et al. Family outbreak of severe pneumonia induced by H7N9 infection. Am J Respir Crit Care Med 2013;188:114-115.

7. Qi X, Qian YH, Bao CJ, et al. Probable person to person transmission of novel avian influenza A (H7N9) virus in Eastern China, 2013: epidemiological investigation. BMJ 2013;347:4752.

8. Liu T, Bi Z, Wang X, et al. One family cluster of avian influenza A (H7N9) virus infection in Shandong, China. BMC Infect Dis 2014;14:98.

9. Li Q, Zhou L, Zhou M, et al. Epidemiology of human infections with avian influenza $\mathrm{A}(\mathrm{H} 7 \mathrm{~N} 9)$ virus in China. N Engl J Med 2014;370:520-532.

10. Cheng VC, Tai JW, Ho SK, et al. Introduction of an electronic monitoring system for monitoring compliance with Moments 1 and 4 of the WHO "My 5 Moments for Hand Hygiene" methodology. BMC Infect Dis 2011;11:151.

11. Cheng VC, Tai JW, Wong LM, et al. Prevention of nosocomial transmission of swine-origin pandemic influenza virus $\mathrm{A} / \mathrm{H} 1 \mathrm{~N} 1$ by infection control bundle. J Hosp Infect 2010;74:271-277.
12. To KK, Song W, Lau SY, et al. Unique reassortant of influenza A (H7N9) virus associated with severe disease emerging in Hong Kong. J Infect 2014;69:60-68.

13. Wang W, Ren P, Mardi S, et al. Design of multiplexed detection assays for identification of avian influenza A virus subtypes pathogenic to humans by SmartCycler real-time reverse transcription-PCR. J Clin Microbiol 2009;47:86-92.

14. Chan KH, To KK, Hung IF, et al. Differences in antibody responses of individuals with natural infection and those vaccinated against pandemic H1N1 2009 influenza. Clin Vaccine Immunol 2011;18:867-873.

15. Ho PL, Sin WC, Chan JF, Cheng VC, Chan KH. Severe influenza A H7N9 pneumonia with rapid virological response to intravenous zanamivir. Eur Respir J 2014;44:535-537.

16. Liu B, Havers F, Chen E, et al. Risk factors for influenza A(H7N9) disease-China, 2013. Clin Infect Dis 2014;59:787-794.

17. Chan KH, To KK, Chan JF, Li CP, Chen H, Yuen KY. Analytical sensitivity of seven point-of-care influenza virus detection tests and two molecular tests for detection of avian origin H7N9 and swine origin $\mathrm{H} 3 \mathrm{~N} 2$ variant influenza A viruses. J Clin Microbiol 2013;51:3160-3161.

18. Cheng VC, Tai JW, Ho YY, Chan JF. Successful control of norovirus outbreak in an infirmary with the use of alcohol-based hand rub. J Hosp Infect 2009;72:370-371.

19. Cheng VC, Wong LM, Tai JW, et al. Prevention of nosocomial transmission of norovirus by strategic infection control measures. Infect Control Hosp Epidemiol 2011;32:229-237.

20. Cheng VC, Tai JW, Chan WM, et al. Sequential introduction of single room isolation and hand hygiene campaign in the control of methicillin-resistant Staphylococcus aureus in intensive care unit. BMC Infect Dis 2010;10:263.

21. Cheng VC, Chan JF, Wong SC, et al. Proactive infection control measures to prevent nosocomial transmission of carbapenemresistant Enterobacteriaceae in a non-endemic area. Chin Med J (Engl) 2013;126:4504-4509.

22. Cheng VC, Tai JW, Chen JH, et al. Proactive infection control measures to prevent nosocomial transmission of vancomycin-resistant enterococci in Hong Kong. J Formos Med Assoc 2014;113:734-741.

23. Xu W, Lu L, Shen B, Li J, Xu J, Jiang S. Serological investigation of subclinical influenza $\mathrm{A}(\mathrm{H} 7 \mathrm{H} 9)$ infection among healthcare and non-healthcare workers in Zhejiang Province, China. Clin Infect Dis 2013;57:919-921.

24. Centers for Disease Control and Prevention. Interim guidance for infection control within healthcare settings when caring for confirmed cases, probable cases, and cases under investigation for infection with novel tnfluenza A viruses associated with severe disease. http://www.cdc.gov/flu/avianflu/h7n9-infection-control. htm. Accessed June 24, 2014.

25. Buxton Bridges C, Katz JM, Seto WH, et al. Risk of influenza A (H5N1) infection among health care workers exposed to patients with influenza A (H5N1), Hong Kong. J Infect Dis 2000;181: 344-348. 